\title{
Triplet Excitation Energy Transfer in Choleic Acid Crystals
}

\author{
Seong-Keun Kook \\ Deparment of Chemistry, Chonnam National University, Gwangiu 500-757, Korea.E-mail: skkook@chonnam.ac.kr \\ Received Angust 9, 2007
}

\begin{abstract}
Time resolved phosphorescence of Dibromobenzophenone (DBBP) choleic acid crystal was observed at $4.2 \mathrm{~K}$ as functions of excitation energy and delay time. The experimental results reveal that the energy transfer efficiency is dependent on the excitation energy, ie the density of acceptors sites. $\Lambda$ s the excitation energy or delay time increases, the resonance phosphorescence does not broaden and shift gradually, rather a broad luminescence band develops about $290 \mathrm{~cm}^{-1}$ to lower energy of the resonance phosphorescence. The observation implies that energy transfer from high to low energy sites in this system is controlled by emission of phonons or vibrons. The data of time resolved experiments were analyzed in terms of a mechanism involving direct donor-acceptor excitation transport by exchange coupling. It was concluded that an isotropic twodimensional exchange interaction topology is consistent with energy transfer in this system.
\end{abstract}

Key Words : Phosphorescence, Energy transfer, Choleic acid. Dibromobenzophenone

\section{Introduction}

Optical luminescence method is one of the most common biological tools to monitor the changes in intracellular ion concentrations. ${ }^{\text {th }}$ Luminescence imaging from a indicator dye injected into a cell provides important insights into the concentration and spatial location of ions throughout single cells. Each dye must be chosen carefully for its ability to provide accurate and reliable information from within a cell. Elements such as toxicity to the cell, self-quenching, intracellular separation, protein binding, and leakage of the dye from the cell frequently complicate the interpretation of the results and must be considered. ${ }^{5-8}$ The minimization of the problems listed above are achieved with employing inert and bio-friendly matrix. A fluorescent indicator dye is entrapped within a protective matrix. The matrix should allow ions to diffuse easily and bind with the indicator but prevents release of indicator dyes into the cell, thus preventing perturbed interactions between the dyes and the cellular contents. Still the entire size should be sufficiently small so as to be minimally invasive and permit rapid response times. Polymers have been used as a typical matrix material. ${ }^{9-11} \mathrm{It}$, however, has distinct disadvantages; limit the number of ions allowed to diffuse and/or cause biological perturbations.

Deoxycholic acid (DCA) is isolated from the bile of animals and forms inclusion compounds by dissolving other molecules. These inclusion compounds, called choleic acids, can be formed with wide variety of guest, polar or non polar, e.g. aliphatic, aromatic, and alicyclic hydrocarbons, alcohols, ketons, fatty acids, esters, ethers, phenols, azodyes. nitriles, peroxides, and amines. ${ }^{12-1+}$ The choleic acid crystals have well defined structures and stoichiometries and sufficiently small disorder that even atoms of the guest molecules can be located by $x$-ray crystallography. ${ }^{15.17}$ Because of this remarkable chemical property, DCA may serve as a host molecule for inert and bio-friendly matrix that has excellent ion selectivity.

Optical properties of choleic acid crystals have been studied previously. ${ }^{18}$ Phosphorescence spectra of 4-methylbenzophenone choleic acid (MBPCA) crystals have been obtained. At lower MBP concentration, the sample appear to consist of choleic acid crystals partially filled with guest, i.e. not all the guest sites are occupied by MBP. This characteristic makes possible to study energy transfer as a function of guest concentration. It, however, was found that energy transfer did not occur at any concentration for the case of MBPCA. The slow rate of energy transfer in MBPCA is likely due to the large intermolecular distance between molecules imposed by the crystal lattice. In order to study further energy transfer in choleic acid, it is necessary to use guest molecules that have a higher intrinsic energy transfer rate. It has been observed that the sensitized phosphorescence of guest molecules containing a heavy atom are substantially stronger than the sensitized phosphorescence of guest molecules of the same moiety without a heavy atom. ${ }^{19-22}$ The sensitized phosphorescence of 4,4'-dibromobenzophenone (DBBP) and 1,4-dibromonaphthalene (DBN) as a dopants in a DCA matrix were observed as a function of the guest concentration. ${ }^{23}$ Direct donor-acceptor triplet excitation energy transfer were characterized with these molecules by using DBBP as the energy donor and DBN as the energy acceptor. The experimental results showed that energy transfer rate increased as the guest concentration increased.

It is of interest to observe the energy transfer in choleic acid crystals as a function of time in order to detemine the spatial distribution of guest molecules in the matrix. Time resolved phosphorescence spectra of DBBP choleic acid were obtained at $4.2 \mathrm{~K}$ as functions of mechanism involving different excitation energy and delay time. The experimental data were analyzed in terms of direct excitation transport by exchange coupling using the theoretical concepts. 


\section{Experimental Section}

DBBP was obtained from ICN Pharmaceuticals and purified by multiple recrystallization $(3 \mathrm{x})$ from ethanol, and zone refined for 100 passes. DCA obtained from Sigma was purified by multiple recrystallization $(4 \mathrm{x})$ from acetonitrile and dried in a vacuum desiccator in order to prevent the formation of an inclusion compound with the solvent. To obtain DBBP choleic acid crystals, an excess of DBBP was mixed with DCA using methanol as a solvent. Crystallization occurred by slow cooling followed by slow evaporation of solvents. After crystallization, the excess DBBP was removed by rinsing with solvent. The stoichiometric ratio of $\mathrm{DBBP}$ to $\mathrm{DCA}$ were detemined to be $2: 1$ by integration of proton NMR spectra of the solution made by dissolving the crystals. These spectra also revealed that the supematant solvent was not occluded in the crystals along with DBBP. The samples were immersed in liquid helium in an optical dewar to achieve a temperature of $4.2 \mathrm{~K}$.

A schematic diagram of the experimental apparatus is shown in Figure 1. The choleic acid crystals were mounted between a quartz plate for phosphorescence spectra measurement. The $\mathrm{S}_{1}$ state was excited by $280 \mathrm{~nm}$ radiation from a $75 \mathrm{~W}$ xenon arc-lamp passed through a monochromator (Jarre] Ash) and glass filter (Coning 7-54). The subsequent steady state phosphorescence was collected through a glass filter (Schott GG 450) to avoid scattered light from excitation, and was monitored with a double spectrometer (Spex 1302 ) and a photomultiplier tube (EMI 9805/474). The photosignal was amplified using a current amplifier at a gain of $10^{\gamma}$ and with a typical time constant of 1 second. As the excitation source for the $T_{1} \leftarrow S_{0}$ transition, a pulsed nitrogen laser (Molectron UV400) and a turable dye laser (Molectron DL400) were used. The nitrogen laser delivers output pulse of $10 \mathrm{~ns}$ in duration, a $0.3 \AA$ in spectral width, and $10 \mathrm{~mW}$ peak power, with turable repetition rate in the range of $10-100 \mathrm{~Hz}$. A boxcar integrator (PAR 162 with model 164 gated integrator modules) was used for timeresolved photosignal acquisition. The same spectrometer

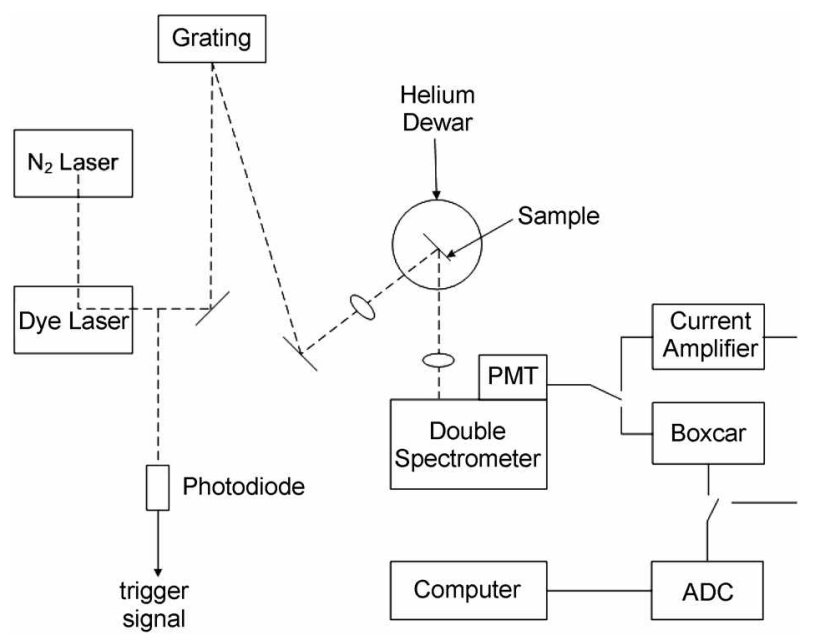

Figure 1. Schematic diagram of instrumentation arrangenent. and photomultiplier tube were used for the time resolved measurement. Light from the dye laser incident on a photodiode was used to trigger the boxcar. The time response of the electronics was tested with a pulse generator. It was found that the response time of the boxcar integrator, including $\mathrm{RC}$ filter on the input, was faster than $\mathrm{I} \mu \mathrm{s}$. The spectra were collected using an analog to digital converter $(\mathrm{ADC})$ and stored on the computer for further analysis.

\section{Experimental Results}

A steady state phosphorescence spectra, obtained at $4.2 \mathrm{~K}$, of DBBP choleic acid is shown in Figure 2. The excitation was at $280 \mathrm{~nm}$. In aromatic carbonyl molecules, the quantum yield of intersystem crossing from the lowest excited state to the lowest excited triplet state is very high, dominating over other decay processes. ${ }^{2+}$ The excited triplet state of DBBP choleic acid is populated by intersystem crossing. The $0-0$ peak of DBBP choleic acid phosphorescence is located at $4300 \AA$, which is a $600 \mathrm{~cm}^{-1}$ red shift relative to the case of DBBP crystalline. The spectral bandwidth of the $0-0$ transition of DBBP choleic acid is approximately $280 \mathrm{~cm}^{-1}$ (fwhi), which is close to that of crystalline DBBP. No Juminescence was detected for DBBP crystalline or pure DCA under the conditions of the experiment. This observation demonstrates that only DBBP cholejc acid exists, not DBBP crystalline.

Figure 3 shows time sampled spectra in the regions of the first vibronic band of the phosphorescence of DBBP choleic acid crystals at $4.2 \mathrm{~K}$ as a function of excitation energy. Excitation wavelength are on and above the high energy edge of the $0-0$ phosphorescence band. In each case the spectrum was sampled after a $10 \mu$ s delay using a $5 \mu \mathrm{s}$ sampling time. In order to avoid interference with scattered light due to the laser, the first vibronic band of the emission was monitored. At the low excitation energy $(4200 \AA)$, two relatively sharp (fwhi $\simeq 40 \mathrm{~cm}^{-1}$ ) resonant emission lines are observed. As the excitation energy increases, a broad (fwhi $\simeq 340 \mathrm{~cm}^{-1}$ ) Juminescence band of increasing intensity develops about $290 \mathrm{~cm}^{-1}$ to lower energy of the resonant

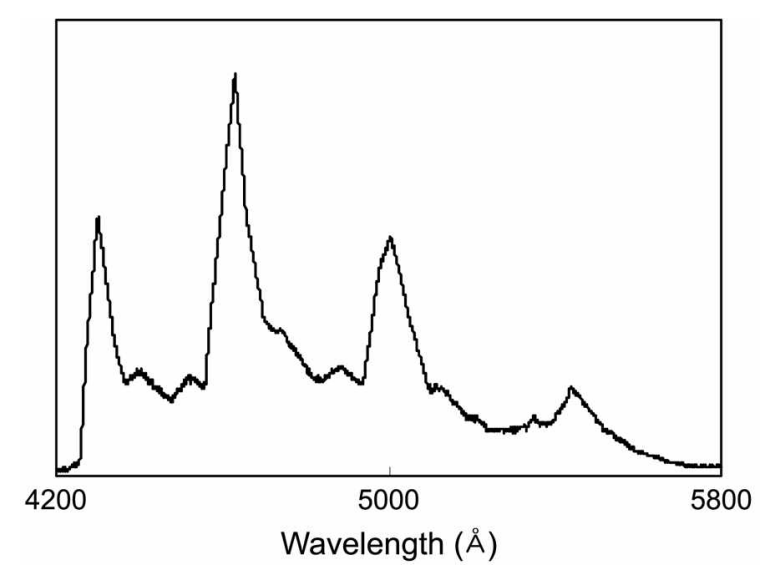

Figure 2. Steady-state phosphorescence obtained at $4.2 \mathrm{~K}$ for DBBP choleic acid crystal. 


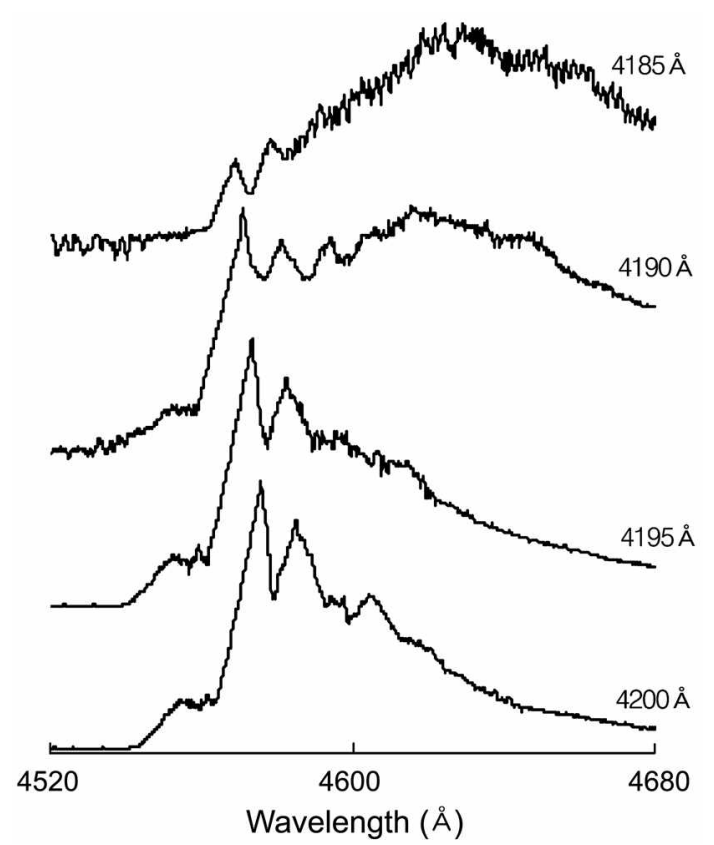

Figure 3. First vibronic band of DBBP choleic acid phosphorescence at $4.2 \mathrm{~K}$ as a function of excitation energy (delay time: 10 is, sampling time: $5 \mu \mathrm{s}$ ).

Table 1. Correlation of excitation and phosphorescence wavelengths ${ }^{t}$

\begin{tabular}{ccccc}
\hline $\begin{array}{l}\lambda_{\text {axe }} \\
(\AA)\end{array}$ & $\begin{array}{c}\lambda_{\text {res }}(0-1) \\
(\AA)\end{array}$ & $\begin{array}{c}\Delta \operatorname{Min} \\
\left(\mathrm{cm}^{-1}\right)\end{array}$ & $\begin{array}{c}\lambda_{x t}(0-1) \\
(\AA)\end{array}$ & $\begin{array}{c}\Delta \nu_{A} \\
\left(\mathrm{~cm}^{-1}\right)\end{array}$ \\
\hline 4180 & 4565 & 1700 & 4640 & 290 \\
4190 & 4570 & 1700 & 4640 & 290 \\
4195 & 4575 & 1700 & - & - \\
4200 & 4580 & 1700 & - & - \\
\hline
\end{tabular}

$\lambda_{x<}$ is the excitation wavelength, $\lambda_{\text {res }}$ is the vibronic resonance phosphorescence shifted by an amount $\Delta r_{i \text { ith, }} \lambda_{\text {ret }}$ is the relaxed

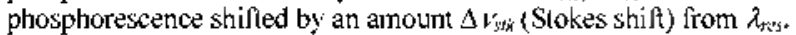

phosphorescence. The resonant emission lines shift with changes in the excitation energy as expected. Table 1 shows the mean position of the doublet, $\lambda_{\text {ress }}$, and the peak position and Stoke shift of the relaxed emission, $\lambda_{\text {ret }}$. The time delay of $10 \mu$ s between excitation and observation assures that the sharp features are resonance phosphorescence and are not caused by Raman scattering. In addition, the decreasing intensity of the sharp features with increasing excitation energy is not characteristic of Raman scattering. The Raman intensity should increase due to the $v^{+}$nonresonant frequency dependence and due to resonant contribution from the $\mathrm{T}_{1} \leftarrow \mathrm{S}_{0}$ absorption.

Time-resolved phosphorescence spectra of DBBP choleic acid at $4.2 \mathrm{~K}$ for different delay times following excitation are shown in Figure 4 . The excitation energy was $4200 \AA$. At a short delay time, e.g. $10 \mu \mathrm{s}$, as monitored above, two sharp resonant emission lines on a broad background are observed. At longer delay times, a broad phosphorescence band develops to lower energy. The intensity of the broad band increases and the intensity of the sharp lines decreases as a

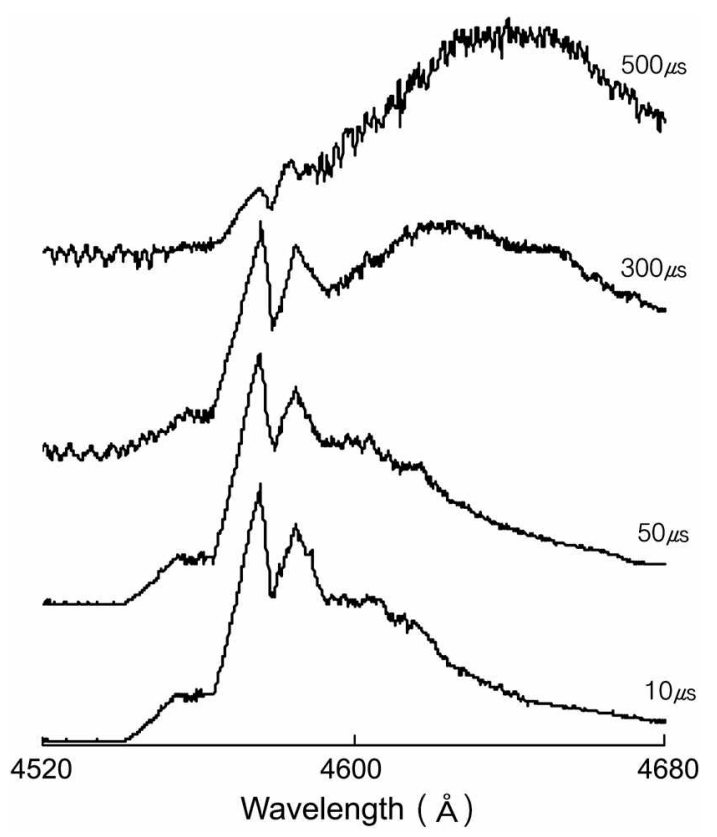

Figure 4. Time-resolved phosphorescence spectra of DBBP choleic acid at $4.2 \mathrm{~K}$ for different delay times with excitation at $4200 \AA$. The delay time is specified, the sampling time is $5 \mu$.

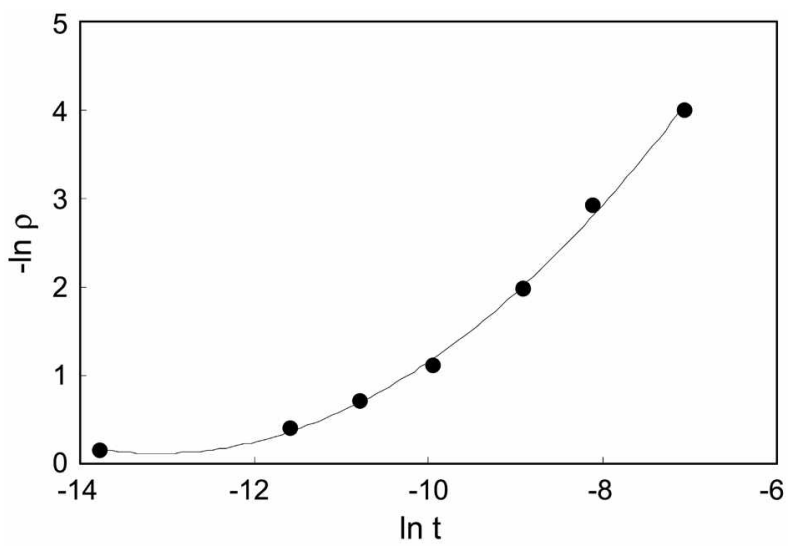

Figure 5. Plot of $\ln x(t)$ vs $\ln t$ for DBBP choleic acid at $4.2 \mathrm{~K}$.

function of the increasing delay time. The position and width of the broad band appear to be independent of the delay time.

\section{Discussion}

Upon excitation, the molecules are divided into three classifications: donor-(molecules requiring an excitation energy equal to the initial excitation energy), acceptor(molecules requiring an excitation energy lower than the initial excitation energy), and barrier- (molecules requiring an excitation energy higher than the intial excitation energy). The number of donors, acceptors, and barriers depends on the initial excitation energy used. As the excitation energy increases, the density of acceptor site increases. This increase would enhance the energy transfer rate from donors to acceptors because the distance between donors and 
acceptors decreases. The experimental results in Figures 3 reveal a strong dependence of the spectral diffusion rate on the excitation energy. At the low excitation energy $(4200 \AA)$, two relatively sharp (fwhi $\simeq 40 \mathrm{~cm}^{-1}$ ) resonant emission lines are observed. As the excitation energy increases resonance phosphorescence does not broaden and shift gradually; rather a broad luminescence peak develops about $290 \mathrm{~cm}^{-1}$ to the red. This jump indicates that energy transfer from high to low energy sites in this system is controlled by emission of phonons or vibrons and not by the density of electronic acceptor sites. When a donor transfer energy to a lower energy acceptor, the excess energy must be dissipated by phonons, which are intermolecular vibrations, or by vibrons, which are intramolecular vibrations. The rate of energy transfer depends upon the density of electronic acceptor states and on the coupling with the phonons and vibrons, as well as on the electronic interaction. Here the term coupling includes the density of phonon and vibron states as well as the strength of the interaction. The density of electronic acceptor states decreases with increasing red shift from the donor. Consequently if this density of states controls the rate, the resonance line will gradually broaden and shift to lower energy. On the other hand, if the phonons and vibrons control the rate, the resonance line will lose intensity and a lower energy band will gain intensity following the time of excitation. The separation between the resonance line and the relaxed band is characteristic of the phonon and/or vibron frequencies that provides significant coupling and high density of states.

We now consider the time evolution of spectral diffusion shown in Figure 4. The excitation is generated at the donor sites by the $T_{1} \leftarrow S_{0}$ absorption, and the acceptor population results only from the energy transfer processes. At $\mathrm{t}=0$, the number of donor sites $N_{0}(0)=N_{0}$, and the excitation density of the acceptor sites $N_{c}(0)=0$. The decay of the excited state population of donor and acceptor molecules can be described by simple kinetics.

$$
\begin{aligned}
& \frac{d N_{d}}{d t}=-k N_{d}-\sum_{j} W\left(r_{i}\right) N_{d} \\
& \frac{d N_{a}}{d t}=-k N_{a}-\sum_{j} W\left(r_{i}\right) N_{d}
\end{aligned}
$$

where $N_{l}$ is the number of donor molecules excited, $k$ is the unimolecular decay rate, and $W\left(r_{i}\right)$ is the rate of energy transfer from the donors to the acceptors at distance $r_{i}, N_{t}$ represents the population of acceptors due to excitation transfer from donors. For the case of triplet energy transfer, the excitation transport generally is govemed by the exchange interaction. The distance dependence of the energy transfer rate between a donor and an acceptor at distance $r$ then is given as ${ }^{25}$

$$
W(r)=k \exp [\gamma(d-r)]
$$

where $d$ is the critical transfer distance, and $\gamma$, which characterizes the range of the exchange interaction, depends on the overlap of the donor phosphorescence and the acceptor absorption spectra. Under the condition of low donor concentration, donor-donor interactions are neglected. Also any possibility of back transfer can be excluded at low temperature.

The donor depopulation function for energy transfer to the acceptor by exchange coupling for all configurations is ${ }^{26,27}$

$$
\Phi(t)=\Pi[1-p(1-\exp [-k t \exp \{\gamma(d-r)\}])]
$$

where $p$ is the probability of a site being occupied by an acceptor. By taking the logarithm and replacing the summation by an integral using continuum approximation for D-dimensional lattice, ${ }^{27}$ equation (4) becomes

$$
\ln \Phi(t)=-V_{D} \rho \gamma^{-D} p g_{D}\left(k t e^{y t}\right)
$$

with

$$
g_{D}(u)=D \int_{0}^{\infty}\left[1-\exp \left(-u e^{-b}\right)\right] v^{D-1} d v
$$

where $V_{D}$ is the volume of a unit sphere in a space of $D$ dimension and $\rho$ is the density of sites. The function $g_{D}(u)$ is given by ${ }^{26}$

$$
\begin{gathered}
g_{1}(u)=\ln u+0.57722 \\
g_{2}(u)=(\ln u)^{2}+1.15443 \ln u+1.9871 \\
g_{3}(u)=(\ln u)^{3}+1.73165(\ln u)^{2}+5.93434 \ln u+5.44487
\end{gathered}
$$

By solving the equation (1) and (2), using the equation (4), the donor and acceptor populations are

$$
\begin{gathered}
N_{d}(t)=N_{0} e^{-k t} \Phi(t) \\
N_{a}(t)=N_{0} e^{-k t}[1-\Phi(t)]
\end{gathered}
$$

Because the intensity of luminescence is proportional to the population, the ratio of the donor intensity to the total intensity at time $t$ can be written as

$$
\rho(t)=\frac{I_{d}(t)}{I_{d}(t)+I_{a}(t)}=\Phi(t)
$$

From eqs. (5) and (12),

$$
\ln \rho(t)=-V_{D} \rho \gamma^{-D} p g_{D}\left(k t e^{y t}\right)
$$

Equations (13) and (7)-(9) show that a plot of $\ln \rho(t)$ as a function of $\ln (t)$ will produce a straight line, a quadratic, or a cubic function for one-, two-, and three-dimensions respectively. In Figure $5, \ln \rho(t)$ versus $\ln (t)$ is plotted for a sample of DBBP choleic acid crystals at $4.2 \mathrm{~K}$. A least-square technique was used to fit the data to a second-order polynomial, the solid curve, consistent with equations (8) and (13). From the agreement between the experimental points and the polynomial function, we may conclude that an isotropic two-dimensional exchange interaction topology is consistent with spectral diffusion in this system. Although the crystal structure of DBBP choleic acid is not known, similar choleic acid ${ }^{14}$ have unit cell dimension of $27 \times 14 \times$ $14 \AA^{3}$, with a nearest neighbor guest-guest distance of $14 \AA^{3}$ along the $b$ axis. For the case of DBBP doped into polymer matrix, ${ }^{23}$ energy transfer takes place at the average distance 
of $19.4 \AA$ which was found from a computer simulation using assumed structure. Considering time resolved experimental results and intermolecular distance between molecules imposed by crystal lattice, it therefore appears that two dimensional exchange interaction topology is consistent with spectral diffusion of triplet excitation energy in DBBP choleic acid crystals.

\section{Conclusions}

Steady state phosphorescence at $4.2 \mathrm{~K}$ is monitored for DBBP choleic acid crystals. The excited triplet state of DBBP choleic acid is populated by intersystem crossing. The $0-0$ peak is $600 \mathrm{~cm}^{-1}$ red shift relative to the case of DBBP crystalline. The spectral bandwidth is $280 \mathrm{~cm}^{-1}$ (fwhi) which is close to that of crystalline DBBP. No luminescence was detected for DBBP crystalline or pure DCA.

Phosphorescence of Dibromobenzophenone (DBBP) choleic acid crystal demonstrated a strong dependence of the spectral diffusion rate on excitation energy and delay time. Time resolved phosphorescence of DBBP choleic acid crystal was observed at $4.2 \mathrm{~K}$ as a function of excitation energy. The results reveal that the energy transfer efficiency is dependent on the number of acceptors. As the excitation energy increases, the resonance phosphorescence does not broaden and shift gradually, rather a broad luminescence band develops $290 \mathrm{~cm}^{-1}$ to lower energy of the resonance phosphorescence. The observation implies that not the density of electronic acceptor states, but the emission of phonons or vibrons controls the energy transfer.

Time evolution of spectral diffusion was observed as a function of delay time. The data were analyzed in terms of a mechanism involving direct donor-acceptor excitation transport by exchange coupling. Consequently, it was concluded that an isotropic two-dimensional exchange interaction topology is consistent with energy transfer in this system.

Acknowledgment. This work was financially supported by Chonnam National University.

\section{References}

1. Slavik, J. Fltorescent Probes in Celltilar and Molectlar Biology;
CRC Press: Boca Ralon, FL, 1994.

2. Mason, W. T. In Biological Technigtes; Sattelle, D. B. Ed.; Academic Press: San Diego, CA, 1993; p 433.

3. Nuccitelli, R. In Methods in Cell Biolog; Wilson, L.; Matsudaira, P., Eds.; Academic Press: San Diego, CA, 1994; vol. 40, p 368.

4. Herman, B. Fhorescence Microscopy, 2 ed.; Springert New York, 1998.

5. Graber, M. L.; DiLillo, D. C.; Friedman, B. L.; Pastoriza-Muniz, E. Antal. Biochem. 1986, $156,202$.

6. CohenKashi, M.; Deusch, M.; Tirosh, R.; Rachmani, H.; Weinreb, A. Spectrochim, Acto Part A 1997, 53, 1655.

7. Overly, C. C.; Lee, K. D.; Berthiaume, E.; Hollenbeck, P. J. Proc. Acad. Sci. U.S.A. 1995, 92, 3156.

8. Brasuel, M. G.; Miller, T. J.; Kopelman, R.; Philbert, M. A. Antahst 2003, $128,1262$.

9. Koo, Y. E.; Cao, Y; Kopelman, R.; Koo, \$. M.; Brasuel, M.; Philbert, M. A. Analyt. Chem, 2004, 76, 2498.

10. Xu, H.; Duck, S. M.; Kopelman, R.; Koo, Y. E. Topics in Fhrorescence Spectroscopy; Geddes, C. D.; Lakowicz, J. R., Eds.; Kluwer, Academic/plenum press: $2005 ; \mathrm{p} 69$.

I1. Morelle, B.; Salmon, J. M.; Vigo, J;; Viallet, P. Cell Biol. Toxicol. $1994,10,339$.

12. Herndon, W. C. J. Chem. Ed, 1967, 44, 724 .

13. Giglio, E. J. Mol, Struct. 1981, 75, 39.

14. Giglio, E. Inchision Compounds, vol 2: Structural Aspect of Inchision Conpothds Forned by Organic Host Lattice; Atwood, J.; Davies, J. E. E.; Macnicol, D. D., Eds.; Academic press: New York, 1984; p 207.

15. Popovitz-Biro, R.; Tang, C. P.; Chang, H. C.; Lahav, M.; Leiserowitz, L. J. Am. Chem, Soc, 1987, 107, 4043 .

16. Tang, C. P.; Chang, H. C.; Popovilz-Biro, R.; Frolow, F.; Lahav, M.; Leiserowitz, L.; Mcmullan, R. K. J. Am. Chen. Soc. 1987. 107,4058 .

17. Chang. H. C.; Popovitz-Biro, R.; Lahav, M.; Leiserowitz, L. $J$. Am. Chem. Soc. 1987, 109, 3883.

18. Kook, S. K.; Kim, D. Y.; Hanson, D. M. Chem. Phys. Lett, 1989, 164,409 .

19. Gobov, G. V.; Konashenko, V. I. Opt. Spectrosc. (USSR) 1977, 43, 622 .

20. Gobov, G. V:; Surin, N. M. Vibronic Spectra of Arontatic Compounds; Smoleusk: Moscow, 1978; 31 .

2I. Gobov, G. V.; Yudenkov, V. V; Yurchenko, A. I, Present-Dar: Aspects of Fine-structure and Selective Spectroscopy; Smoleusk: Moscow, 1984; p 199.

22. Gobov, G. V; Yudenkov, V. V; Savchenkov, V. I. Opt. Spectrosc. (CSSR) 1986, 60, 452.

23. Kook, S. K.; Hanson, D. M. J. Phys. Chem. 1993, $97,9578$.

24. Lin, Y,; Hanson, D. M. Phys. Chem, 1987, 91, 2279.

25. Dexter, D. L. J. Chem. Phys, $1953,21,836$.

26. Blumen, A. J. Chem. Phss. 1980, 72, 2632.

27. Blumen, A.; Manz, J. J. Chem. Phys. 1979, 7I, 4694. 